\title{
RARE OCCURRENCE OF LOBULAR CAPILLARY HAEMANGIOMA IN MAXILLARY POSTERIOR SEGMENT INVOLVING THE ALVEOLAR MUCOSA: ACASE REPORT
}

\author{
${ }^{1}$ Suraj Pandey, ${ }^{2}$ AshishSaini, ${ }^{3}$ Sunil Chandra Verma, ${ }^{4}$ Pranav Kumar Singh \\ ${ }^{1-4}$ Department of Periodontology, \\ Babu Banarasi Das College of Dental Sciences, Lucknow
}

\begin{abstract}
:
Pyogenic granuloma (PG) is one of the inflammatory hyperplasias seen in the oral cavity. This term is a misnomer because the lesion is unrelated to infection and in reality arises in response to various stimuli such as low-grade local irritation, traumatic injury or hormonal factors. PG is predominantly seen in the second decade of life in young adult females. PGs generally are soft, painless, and deep red to reddish-purple in color. It shows a striking predilection for maxillary anterior area. Also, majority of PGs are found on the marginal gingiva with only $15 \%$ of the tumors on the alveolar part. There are two kinds of PG namely lobular capillary hemangioma ( $\mathrm{LCH}$ type) and non-LCH type, which manifest as distinct entities and differ in many aspects. Although excisional surgery is thetreatment of choice for it, some other treatmentprotocols such as the use of Nd:YAG laser, flash lamppulsed dye laser, cryosurgery, intralesional injection ofethanol or corticosteroid and sodium tetradecylsulfatesclerotherapy have been proposed.We hereby present a rare case report of lobular capillary hemangioma occurring in the maxillary posterior region. Also, contrary to its site specificity, it involved the attached gingiva and alveolar mucosa, thereby presenting a diagnostic dilemma. The various differences between the LCH and Non-LCH type PG are also discussed emphasising their different pathways of evolution.
\end{abstract}

\author{
Key words: \\ capillary haemangioma, \\ Non-LCH pyogenic \\ granuloma, reactive \\ hyperplasias \\ Source of support : Nil
Conflict of interest: None
}

INTRODUCTION: Soft tissue enlargements of the oral cavity often present a diagnostic challenge to the clinician because a diverse group of pathologic processes can produce such lesions. Such lesions may represent a variation of normal anatomic structures, inflammation, cysts, developmental anomalies and neoplasm. Within this group is a category of reactive hyperplasias, which include epulisfissuratum, palatal papillary hyperplasia, fibroma, giant cell granuloma, pregnancy epulis and pyogenic granuloma (PG).[1]

PG is a common tumor-like growth of the oral cavity or skin that is considered to be non-neoplastic in nature. It has been referred to in literature by a variety of other names such as granuloma pediculatumbenignum, vascular epulis, Crocker and Hartzell's disease.[2] The term "pyogenic granuloma" is a misnomer because the lesion does not contain pus and is not strictly speaking a granuloma. ${ }^{1}$ There are two kinds of PG namely lobular capillary hemangioma (LCH type) and nonLCH type, which differ in their histological features. ${ }^{3}$ Gingiva, lips, tongue and buccal mucosa are the common sites of oral PG. It shows a striking predilection for maxillary anterior area.[4] According to Vilmann et al, majority of PGs are found on the marginal gingiva with only $15 \%$ of the tumors on the alveolar part.[5]

We hereby present a rare case report of long standing lobular capillary hemangioma occurring in the maxillary posterior region. Also, contrary to its site specificity, it involved the attached gingiva and alveolar mucosa, thereby posing a diagnostic dilemma.

\section{CASE REPORT:}

A 29 year old female reported to the dental clinic, with the chief complaint of a growth in the maxillary right posterior 
region. Growth appeared eight years back and has been slowly growing to attain the present size (Figure 1).

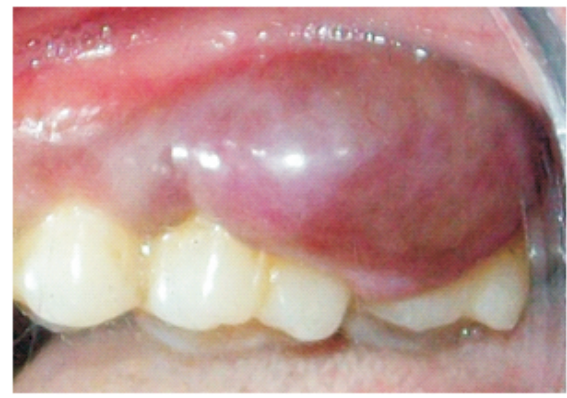

Figure 1- Pre-Operative Picture depicting growth in maxillary posterior region

Mild intermittent pain was associated with the growth which increased on chewing food. Patient reported difficulty in mastication and was also concerned for the nature of the lesion.

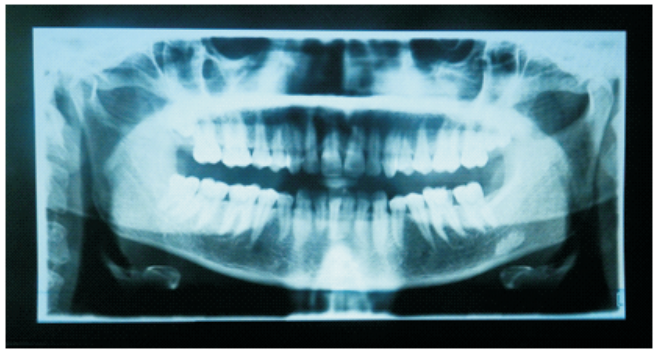

Figure 2- Orthopantomogramm depicting no significant bone loss

Due to the long history and fluctuant nature of the lesion, FNAC was performed for the patient before any surgical intervention. Fluid was collected from the centre of the lesion and was sent for analysis (Figure 3,4).

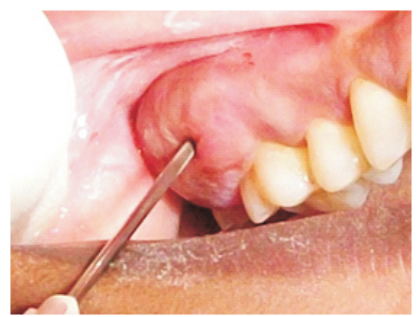

Figure 3- FNAC being performed

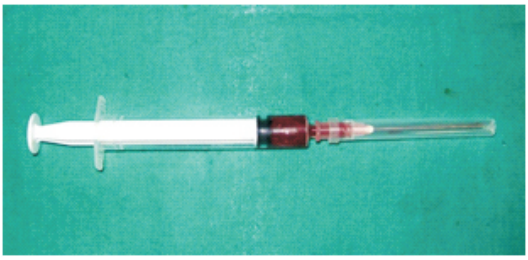

Figure 4- Aspirated FNAC fluid
Report showed the fluid to primarily contain RBCs and few inflammatory cells. Based on its clinical signs and symptoms, a differential diagnosis consisted of PG, peripheral giant cell granuloma, peripheral ossifying fibroma, metastatic carcinoma, capillary hemangioma and Kaposi's sarcoma[1]. Surgical excision was planned for the patient and electrocauteryunit was kept at hand anticipating postoperative haemorrhage. Under local anesthesia, the growth was probed to check its bleeding tendency. Afterwards, an excisional biopsy was performed with a wide margin down to the periosteum with curettage was performed (Figure 5).

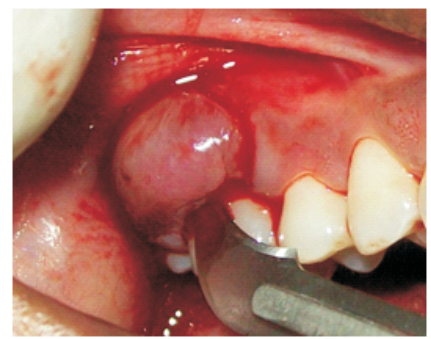

Figure 5-Excisional biopsy of lesion

The excised lesion measuring $2 \mathrm{~cm} \mathrm{X} 1$ cmwas sent for histopathological examination (Figure 6).

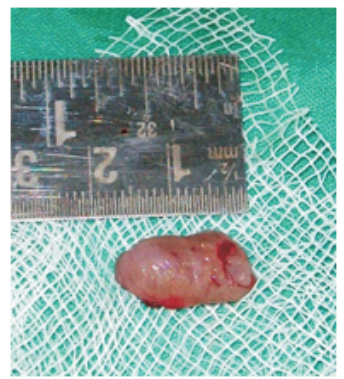

Figure 6-Excised lesion

Patient was kept under antibiotics and analgesicsand was recalled for a post-operative check-up after $48 \mathrm{hrs}$. No complaints were reported by the patient. The healing was satisfactory. Patient was kept on a follow up for one year and no recurrence was seen (Figure 7

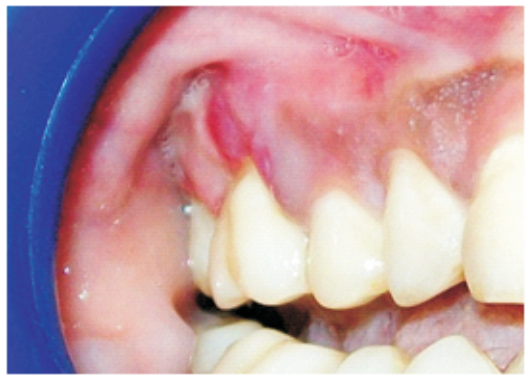

Figure 7- One week Post-Operative 
Histopathological examination showed vascular proliferations that were organised in lobular aggregates. Numerous endothelium lined vascular channels were seen filled with RBCs. A mixed inflammatory cell infiltrate of neutrophils, plasma cells and lymphocytes were visualised in the stroma. The overlying epithelium was stratified squamous type with flattened rete ridges (Figure $8 \& 9$ ). Therefore a final diagnosis of Lobular Capillary Haemangioma was rendered.

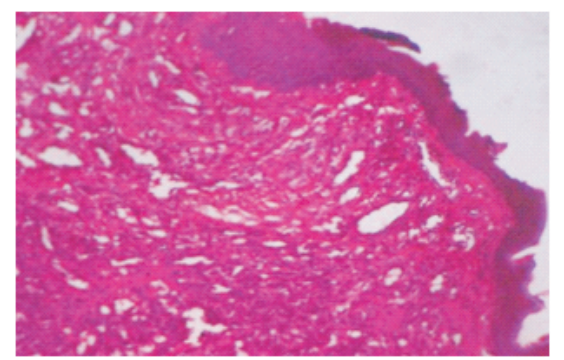

Figure 8- Numerous vascular channels in a densely inflamed CT stroma with overlying stratified squamous epithelium (4X magnification)

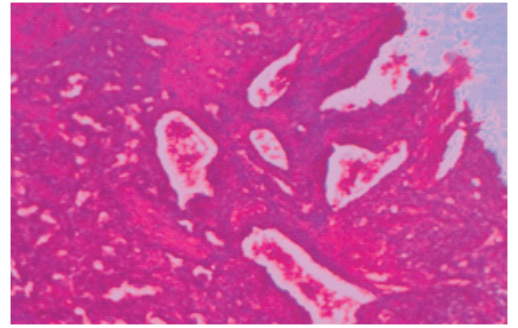

Figure 9- Endothelium lined vascular channels filled with RBCs (10X magnification)

\section{DISCUSSION:}

Occurrence of pyogenic granuloma in man was first described in 1897 by Poncet and Dor. At that time, it was called botryomycosis hominis.[2] However according to some

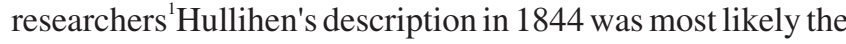
first PG reported in English literature and the term "pyogenic granuloma" or granuloma pyogenicum" was introduced by Hartzell in 1904.Angelopoulos AP proposed the term "hemangiomatous granuloma" that accurately expresses the histopathologic picture (hemangioma like) and the inflammatory nature (granuloma) of oral pyogenic granuloma.[6]

Although it is a common disease in the skin, it is extremely rare in the gastrointestinal tract, except for the oral cavity where it is often found on keratinized tissue. ${ }^{1}$ Bhaskaret al. in their study observed that oral pyogenic granuloma comprized about $1.85 \%$ of all oral pathoses, other than caries and gingivitis treated at US Army Institute of Dental Research. While some investigators regard PG as a benignneoplasm, it is usually considered to be a reactive tumorlikelesion which arises in response to various stimulisuch as a chronic lowgrade local irritation, traumatic injury, hormonal factors or certain kinds of drugs. Although it was originally thought to be caused by pyogenic organisms, it is now believed to be unrelated to infection.[1] Some factors such as inducible nitric oxide synthase, vascular endothelial growth factor, basic fibroblast growth factor or connective tissue growth factorare known to be involved in angiogenesis and rapid growth of PG.[1] Sangueza and Requena stated that pyogenic granuloma lesions express factor VIII - related antigen positivity in the endothelial cells lining large vessels, but are negative in the cellular areas.[2]

Although PG may occur in all ages, it is predominant in the second decade of life in young adult females. $\left[{ }^{7]}\right.$ Intraorally, it can present with a wide array of clinical appearances, ranging from a sessile lesion to an elevated mass. Pyogenic granulomas generally are soft, painless, and deep red to reddish-purple in color.[2] Radiographic findings are absent in pyogenic granuloma. However, angelopoulos AP in his review observed that localized alveolar bone resorption in rare instances of large and long standing gingival tumors can be seen.[6] PG of the gingiva develops in up to 5\% of pregnancies, hence the terms "pregnancy tumor" and "granuloma gravidarum" are often used.[8] Generally it appears in the $2 \mathrm{nd}$ - 3rd month of pregnancy, with a tendency to bleed and a possible interference with mastication.

There are two histological types of PG. The first type is characterized by proliferating blood vessels that are organized in lobular aggregates (LCH type), whereas the second type (non-LCH type) consists of highly vascular proliferation that resembles granulation tissue. ${ }^{1}$ Epivatianos et al. observed that the two types of PG were clinically different in that LCH type of PG occurred more frequently $(66 \%)$ as a sessile lesion, whereas non-LCH type of PG mostly occurred as a pedunculatedone (77\%). He also observed that foci of fibrous maturation were present in $15 \%$ of non-LCH type of PG but were totally absent in LCH type of PG.[3] In the central area of non- LCH type of PG, a significantly greater number of vessels with perivascular mesenchymal cells nonreactive for -smooth muscle actin and muscle-specific actin is present than in LCH type of PG. ${ }^{1}$ Toida et al. found that the presence of blood vessels with different luminal diameter in the lobular area of LCH type of PG and in the central area of 
non-LCH type of PG may be because different pathogenic factors influence their development.[9]

Surgical excision is the treatment of choice.[2] After surgicalexcision of gingival lesions, curettage of underlying tissue isrecommended.[10] Some new approaches for treatment such as cryosurgery, excision by Nd:YAG laser, flash lamp pulsed dye laser, injection of ethanol or corticosteroid and sodium tetradecylsulfatesclerotherapy have also been reported as alternative therapies. ${ }^{1}$ Bhaskar and Jacoway has reported recurrence rate of $15.8 \%$ after conservative excision. Vilmannet al. observed that gingival cases show a much higher recurrence rate than lesions from other oral mucosal sites.[2]

\section{CONCLUSION:}

This case report demonstrates an unusual site of occurrence of $\mathrm{LCH}$ in the oral cavity. It also emphasiseson the two histological variants of PG: LCH type and Non-LCH type. The differences between these two suggest that the two types of $\mathrm{PG}$ represent distinct clinical entities and may have different pathways of evolution.

\section{IREFERENCES:}

1. Jafarzadeh H, Sanatkhani M, Mohtasham N. Oral pyogenic granuloma: a review. J Oral Sci 2006;48:167-175.

2. Kamal R, Dahiya P, Puri A. Oral pyogenic granuloma: Various concepts of etiopathogenesis. J Oral MaxillofacPathol 2012;16:79-82.

3. Epivatianos A, Antoniades D, Zaraboukas T, Zairi E, Poulopoulos A, KiziridouA et al. Pyogenic granuloma of the oral cavity: comparative study of its clinicopathological and immunohistochemical features. PatholInt2005;55:391-397.

4. Neville BW, Damm DD, Allen CM, Bouquot JE. Oral \& maxillofacial pathology. 2nd ed. Philadelphia: WB Saunders;2002. p. 437-495.

5. Vilmann A, Vilmann P, Vilmann H. Pyogenic granuloma: evaluation of oral conditions. $\mathrm{Br} \mathrm{J}$ Oral MaxillofacSurg 1986; 24: 376-382.

6. Angelopoulos AP. Pyogenic granuloma of the oral cavity: Statistical analysis of its clinical features. J Oral Surg 1971;29:840-7.
7. Al-Khateeb T, Ababneh K. Oral pyogenic granuloma in Jordanians: a retrospective analysis of 108 cases. J Oral MaxillofacSurg 2003; 61: 1285-1288.

8. Sills ES, Zegarelli DJ, Hoschander MM, Strider WE. Clinical diagnosis and management of hormonally responsive oral pregnancy tumor (pyogenic granuloma). J Reprod Med 1996;41: 467-47.

9. Toida M, Hasegawa T, Watanabe F, Kato K, Makita H, Fujitsuka H et al. Lobular capillary hemangioma of the oral mucosa: clinicopathological study of 43 cases with a special reference to immunohistochemical characterization of the vascular elements. PatholInt2003;53:1-7.

10. Patil K, Mahima VG, Lahari K. Extragingival pyogenic granuloma. Indian $J$ Dent Res 2006;17:199-202.

\section{CORRESPONDING AUTHOR:}

\section{Dr. Suraj Pandey}

3/299, Vishal Khand, Gomti Nagar, Lucknow

Email :drsurajpandey9@gmail.com 